\title{
Orthogonal polynomial approach to fluids with internal degrees of freedom: The case of nonpolar, polarizable molecules
}

\author{
F. Lado \\ Department of Physics, North Carolina State University, Raleigh, North Carolina 27695-8202
}

(Received 26 August 1996)

\begin{abstract}
The molecules of real liquids have internal degrees of freedom that may couple with the external ones of position and orientation so that they affect and are affected by the microscopic liquid structure. For cases where the internal coordinates possess a Boltzmann-like distribution, a procedure is described whereby such coordinates can be incorporated into the conventional formulation of classical liquid theory with no approximations beyond some reliable closure relation familiar from simple liquids. The basis of the procedure is expansions in appropriate orthogonal polynomials of the internal coordinates. This program is successfully carried out for a classical model of nonpolar, polarizable molecules treated as Drude oscillators, generalizing published solutions of the mean spherical approximation for the same model. [S1063-651X(97)05701-2]
\end{abstract}

PACS number(s): 61.20.Gy, 61.25.Em, 77.22.Ch

\section{INTRODUCTION}

The theory of liquids has focused strongly since its inception on the averaged geometrical structure of liquids embodied in the pair distribution function as the key feature needed to predict thermodynamic and scattering data for these systems. In this scheme, the molecules of the liquid are usually treated as inert and featureless, apart from assumed fixed shapes, a simplification that has had great success indeed, to the extent that even a billiard ball model of atoms as hard spheres has relevance for real liquids. The pertinent degrees of freedom are then the fluctuating positions of the centers of mass of the molecules and, for nonspherical potentials, their fluctuating orientations, all treated in classical terms. In reality, of course, the internal structure of molecules is dynamically altered by the changing configurations of their many near neighbors in a condensed state (one has only to think of the continuous emission spectra of heated solids and liquids versus the line spectra of their vapors) and in turn these internal changes feed back into the averaged structure through fluctuations in the effective intermolecular potentials. Changes in the internal structure of the molecules involve the electrons, whose small mass means shorter time scales and longer de Broglie wavelengths requiring a quantum-mechanical description. Much work has already been done in this intermediate domain where the internal and external degrees of freedom are coupled to each other, as described in a recent review by Stratt [1]. The mean spherical approximation and other linearized approximations of liquid state theory figure prominently in this work, as does good physical insight to carry through the calculations. This paper is motivated by the optimistic conjecture that these are not necessary features and that internal degrees of freedom can be absorbed into the "canonical" formulation of classical liquid state theory in much the same manner as orientational degrees of freedom have been - through the use of appropriate orthogonal polynomial expansions - so they can be dealt with using established procedures demanding no unusual insight.

It is hardly possible to prove or disprove this proposition in the fashion of a mathematical theorem; experience on a case-by-case basis will decide. In this paper [2], we have chosen probably the simplest example of coupled internal and external degrees of freedom in a fluid: nonpolar, polarizable molecules modeled as classical Drude oscillators. (The significant complications that are added by a quantummechanical description of the internal degrees of freedom are not dealt with here.)

Molecules lacking a permanent dipole moment will nonetheless display instantaneous moments $\mathbf{p}$ that fluctuate randomly within some overall constraints and so will interact through dipole-dipole forces with other such molecules. We will follow Pratt [3] and Høye and Stell [4] in assuming an intrinsic thermal distribution of Gaussian form

$$
f_{0}(p)=\frac{1}{\left(2 \pi \alpha_{0} / \beta\right)^{3 / 2}} \exp \left(-\frac{\beta p^{2}}{2 \alpha_{0}}\right)
$$

for the spontaneous dipole moment $\mathbf{p}$ of an isolated molecule, where $\alpha_{0}$ is the polarizability of the molecule and $\beta=1 / k_{B} T$, with $T$ the Kelvin temperature and $k_{B}$ Boltzmann's constant. These authors have independently solved the mean spherical approximation (MSA) for this model (with inclusion of a permanent dipole in the case of the Høye-Stell work) and find that the fluctuating dipole distribution $f(p)$ in the dense liquid remains Gaussian, an inherent restriction of the MSA, with a larger polarizability $\alpha>\alpha_{0}$. In this paper, we generalize these solutions to permit use of any closure found accurate in studies of simple liquids and formulate an exact, computable expression for the distribution $f(p)$. By going beyond a Gaussian restriction we can show by calculation that in fact the Gaussian shape in this case is actually an excellent approximation. The key to these generalizations is expansion in polynomials of $p$ that are orthogonal with weight function $f(p)$.

\section{DISTRIBUTION FUNCTIONS}

\section{A. Preliminaries}

We consider a system of $N$ molecules, each with intrinsic polarizability $\alpha_{0}$ and fluctuating polarity but no permanent 
dipole moment, contained in a volume $V$ at temperature $T$. In the presence of an external electric field $\mathbf{E}^{0}(\mathbf{r})$, the configurational partition function of the system is

$$
\begin{aligned}
Z= & \frac{1}{\left(2 \pi \alpha_{0} / \beta\right)^{3 N / 2} V^{N}} \int \prod_{j=1}^{N}\left(d \mathbf{r}_{j} d \mathbf{p}_{j}\right) \\
& \times \exp \left[-\beta \sum_{j=1}^{N}\left(\frac{p_{j}^{2}}{2 \alpha_{0}}-\mathbf{p}_{j} \cdot \mathbf{E}_{j}^{0}\right)-\beta U_{D D}-\beta U_{0}\right],
\end{aligned}
$$

where $\beta=1 / k_{B} T$ and

$$
\begin{gathered}
\mathbf{E}_{j}^{0}=\mathbf{E}^{0}\left(\mathbf{r}_{j}\right)=\int d \mathbf{r} \mathbf{E}^{0}(\mathbf{r}) \delta\left(\mathbf{r}-\mathbf{r}_{j}\right), \\
U_{D D}=\sum_{i<j} u\left(\mathbf{r}_{i j}, \mathbf{p}_{i}, \mathbf{p}_{j}\right), \\
U_{0}=\sum_{i<j} u_{0}\left(r_{i j}\right) .
\end{gathered}
$$

Here $u$ is the usual dipole-dipole interaction

$$
u\left(\mathbf{r}, \mathbf{p}_{1}, \mathbf{p}_{2}\right)=-\frac{1}{r^{3}}\left[3\left(\hat{\mathbf{r}} \cdot \mathbf{p}_{1}\right)\left(\hat{\mathbf{r}} \cdot \mathbf{p}_{2}\right)-\mathbf{p}_{1} \cdot \mathbf{p}_{2}\right],
$$

where $\hat{\mathbf{r}}$ is the unit vector in the $\mathbf{r}$ direction, while for $u_{0}$ we take the Lennard-Jones potential

$$
u_{0}(r)=4 \epsilon_{\mathrm{LJ}}\left[\left(\frac{\sigma}{r}\right)^{12}-\left(\frac{\sigma}{r}\right)^{6}\right]
$$

The one- and two-body distribution functions are defined by the canonical ensemble averages

$$
\begin{aligned}
\rho^{(1)}(\mathbf{r}, \mathbf{p})= & \left\langle\sum_{j=1}^{N} \delta\left(\mathbf{r}-\mathbf{r}_{j}\right) \delta\left(\mathbf{p}-\mathbf{p}_{j}\right)\right\rangle, \\
\rho^{(2)}\left(\mathbf{r}, \mathbf{p}, \mathbf{r}^{\prime}, \mathbf{p}^{\prime}\right)= & \left\langle\sum_{i \neq j} \delta\left(\mathbf{r}-\mathbf{r}_{i}\right) \delta\left(\mathbf{p}-\mathbf{p}_{i}\right) \delta\left(\mathbf{r}^{\prime}-\mathbf{r}_{j}\right)\right. \\
& \left.\times \delta\left(\mathbf{p}^{\prime}-\mathbf{p}_{j}\right)\right\rangle .
\end{aligned}
$$

In the absence of an external field, $\mathbf{E}^{0} \rightarrow \mathbf{0}$, the system is uniform and isotropic and these functions simplify to

$$
\begin{gathered}
\rho^{(1)}(\mathbf{r}, \mathbf{p})=\rho f(p), \\
\rho^{(2)}\left(\mathbf{r}, \mathbf{p}, \mathbf{r}^{\prime}, \mathbf{p}^{\prime}\right)=\rho^{2} f(p) f\left(p^{\prime}\right) g\left(\mathbf{r}-\mathbf{r}^{\prime}, \mathbf{p}, \mathbf{p}^{\prime}\right),
\end{gathered}
$$

where $\rho=N / V$ is the density. The calculations in Sec. IV for the distribution $f(p)$ of the fluctuating dipoles in the dense liquid and for the pair distribution function $g\left(\mathbf{r}, \mathbf{p}, \mathbf{p}^{\prime}\right)$, as well as the remainder of the discussion in this section, will be for zero external field. The external field is used in Sec. III to formulate the dielectric properties of the system.
The two distribution functions are of course coupled by the intermolecular potential $u$. A direct expression of this coupling is obtained by differentiating $f(p)$ with respect to $p$ to yield

$$
\frac{d}{d p} \ln \left[\frac{f(p)}{f_{0}(p)}\right]=-\rho \int d \mathbf{r} d \mathbf{p}^{\prime} f\left(p^{\prime}\right) g\left(\mathbf{r}, \mathbf{p}, \mathbf{p}^{\prime}\right) \beta u\left(\mathbf{r}, \hat{\mathbf{p}}, \mathbf{p}^{\prime}\right),
$$

the first member of a Kirkwood-Born-Green-Yvon hierarchy [5]. Note the unit vector $\hat{\mathbf{p}}$ in the potential $u$ of this expression; the dependence on $p$ on the right-hand side is carried entirely by $g$.

Calculation of $f(p)$ from Eq. (12) requires knowing the pair distribution function (PDF). In classical liquid state theory, the PDF is obtained from the Ornstein-Zernike equation and an approximate closure relation. The first of these, the Ornstein-Zernike (OZ) equation, generalized for fluctuating dipoles, reads [6]

$$
\begin{aligned}
h\left(\mathbf{r}_{12}, \mathbf{p}_{1}, \mathbf{p}_{2}\right)= & c\left(\mathbf{r}_{12}, \mathbf{p}_{1}, \mathbf{p}_{2}\right)+\rho \\
& \times \int d \mathbf{r}_{3} d \mathbf{p}_{3} f\left(p_{3}\right) h\left(\mathbf{r}_{13}, \mathbf{p}_{1}, \mathbf{p}_{3}\right) c\left(\mathbf{r}_{32}, \mathbf{p}_{3}, \mathbf{p}_{2}\right),
\end{aligned}
$$

where $h\left(\mathbf{r}, \mathbf{p}_{1}, \mathbf{p}_{2}\right) \equiv g\left(\mathbf{r}, \mathbf{p}_{1}, \mathbf{p}_{2}\right)-1$ and $c\left(\mathbf{r}, \mathbf{p}_{1}, \mathbf{p}_{2}\right)$ is a new unknown, the direct correlation function (DCF). The second, or closure, relation almost expresses the DCF back in terms of the PDF and the system's interactions [6],

$$
\begin{aligned}
c\left(\mathbf{r}, \mathbf{p}_{1}, \mathbf{p}_{2}\right)= & h\left(\mathbf{r}, \mathbf{p}_{1}, \mathbf{p}_{2}\right)-\ln g\left(\mathbf{r}, \mathbf{p}_{1}, \mathbf{p}_{2}\right)-\beta u_{0}(r) \\
& -\beta u\left(\mathbf{r}, \mathbf{p}_{1}, \mathbf{p}_{2}\right)+B\left(\mathbf{r}, \mathbf{p}_{1}, \mathbf{p}_{2}\right) .
\end{aligned}
$$

While a formal expression in terms of diagram summations is known for $B$, the so-called bridge function, it is of little practical use and this function is usually approximated in a more-or-less ad hoc fashion, giving rise to the variety of closures in use today; for purposes of the present discussion, $B$ will be treated as a given.

Manifestly, solving the coupled (OZ plus closure) equations for $g\left(\mathbf{r}, \mathbf{p}_{1}, \mathbf{p}_{2}\right)$ in turn requires knowing the dipole distribution function $f(p)$. An iterative procedure will evidently be needed.

Equations (12)-(14) (plus an approximation for $B$ ) constitute a closed set whose solution gives detailed microscopic information sufficient to determine all the thermodynamic and electrostatic properties of the system. The equations being nonlinear, "solution" here generally means "numerical solution." As they appear, however, these equations are too complex for numerical handling, since quantities such as $g\left(\mathbf{r}, \mathbf{p}_{1}, \mathbf{p}_{2}\right)$ are each functions of six variables: $r, p_{1}, p_{2}$, and $\omega_{1}, \omega_{2}$, the directions of $\mathbf{p}_{1}, \mathbf{p}_{2}$, for which three Euler angles suffice. It has long been standard practice in the study of molecular fluids [7] to reduce the complexity of the angular description by using expansions in spherical harmonics $Y_{l m}(\omega)$ [8]. It is a small but fruitful step to extend this practice to the internal coordinate description as well, using expansions in an appropiate set of orthogonal polynomials $Q_{n l}(p)$. This is done in the Sec. II B. We note first that, as usual, Eqs. (13) and (14) are more conveniently handled by 
reassigning the unknown to be the indirect correlation function $\gamma=h-c$, rather than $g$, and rewriting Eq. (13) in Fourier transform representation. The result is

$$
\begin{aligned}
\widetilde{\gamma}\left(\mathbf{k}, \mathbf{p}_{1}, \mathbf{p}_{2}\right)= & \rho \int d \mathbf{p}_{3} f\left(p_{3}\right)\left[\widetilde{\gamma}\left(\mathbf{k}, \mathbf{p}_{1}, \mathbf{p}_{3}\right)\right. \\
& \left.+\widetilde{c}\left(\mathbf{k}, \mathbf{p}_{1}, \mathbf{p}_{3}\right)\right] \widetilde{c}\left(\mathbf{k}, \mathbf{p}_{3}, \mathbf{p}_{2}\right)
\end{aligned}
$$

for the $\mathrm{OZ}$ equation (13) and

$$
\begin{aligned}
c\left(\mathbf{r}, \mathbf{p}_{1}, \mathbf{p}_{2}\right)= & \exp \left[-\beta u_{0}(r)-\beta u\left(\mathbf{r}, \mathbf{p}_{1}, \mathbf{p}_{2}\right)+\gamma\left(\mathbf{r}, \mathbf{p}_{1}, \mathbf{p}_{2}\right)\right. \\
& \left.+B\left(\mathbf{r}, \mathbf{p}_{1}, \mathbf{p}_{2}\right)\right]-1-\gamma\left(\mathbf{r}, \mathbf{p}_{1}, \mathbf{p}_{2}\right)
\end{aligned}
$$

for the closure (14). The PDF is then computed from $\gamma$ as

$$
\begin{aligned}
g\left(\mathbf{r}, \mathbf{p}_{1}, \mathbf{p}_{2}\right)= & \exp \left[-\beta u_{0}(r)-\beta u\left(\mathbf{r}, \mathbf{p}_{1}, \mathbf{p}_{2}\right)+\gamma\left(\mathbf{r}, \mathbf{p}_{1}, \mathbf{p}_{2}\right)\right. \\
& \left.+B\left(\mathbf{r}, \mathbf{p}_{1}, \mathbf{p}_{2}\right)\right] .
\end{aligned}
$$

We remark for completeness that a transform pair $f(\mathbf{r})$ and $\widetilde{f}(\mathbf{k})$ is defined by

$$
\begin{gathered}
f(\mathbf{r})=\frac{1}{(2 \pi)^{3}} \int d \mathbf{k} \widetilde{f}(\mathbf{k}) e^{i \mathbf{k} \cdot \mathbf{r}}, \\
\widetilde{f}(\mathbf{k})=\int d \mathbf{r} f(\mathbf{r}) e^{-i \mathbf{k} \cdot \mathbf{r}} .
\end{gathered}
$$

\section{B. Expansions in orthogonal polynomials}

The conventional next step in solving Eqs. (15) and (16) is, as noted, to explicitly break out the angular dependence of all functions in the form of expansions in spherical harmonics [7], writing, e.g., [9]

$$
\begin{aligned}
\gamma\left(\mathbf{r}, \mathbf{p}_{1}, \mathbf{p}_{2}\right) & =\gamma\left(r, p_{1}, p_{2}, \omega_{1}, \omega_{2}\right) \\
& =4 \pi \sum_{l_{1}, l_{2}, m} \gamma_{l_{1} l_{2} m}\left(r, p_{1}, p_{2}\right) Y_{l_{1} m}\left(\omega_{1}\right) Y_{l_{2} \bar{m}}\left(\omega_{2}\right),
\end{aligned}
$$

where $\bar{m}=-m$. In this and similar expressions, the vector $\mathbf{r}$ has been implicitly chosen as the $z$ direction in the specification of the Euler angles $\omega=(\theta, \phi)$. What makes this expansion particularly useful [10] is of course the orthogonality of the spherical harmonics,

$$
\int d \omega Y_{l m}(\omega) Y_{l^{\prime} m^{\prime}}^{*}(\omega)=\delta_{l l^{\prime}} \delta_{m m^{\prime}},
$$

so that the coefficients of the expansion are immediately obtainable by quadratures,

$$
\begin{aligned}
\gamma_{l_{1} l_{2} m}\left(r, p_{1}, p_{2}\right)= & \frac{1}{4 \pi} \int d \omega_{1} d \omega_{2} \gamma\left(r, p_{1}, p_{2}, \omega_{1}, \omega_{2}\right) \\
& \times Y_{l_{1} m}^{*}\left(\omega_{1}\right) Y_{l_{2} \bar{m}}^{*}\left(\omega_{2}\right) .
\end{aligned}
$$

We now similarly break out the fluctuating-dipole dependence in the form of expansions in polynomials of $p$,

$$
\gamma_{l_{1} l_{2} m}\left(r, p_{1}, p_{2}\right)=\sum_{n_{1}, n_{2}} \gamma_{l_{1} l_{2} m}^{n_{1} n_{2}}(r) Q_{n_{1} l_{1}}\left(p_{1}\right) Q_{n_{2} l_{2}}\left(p_{2}\right),
$$

which are constructed to be orthogonal with weight function $f(p)$,

$$
4 \pi \int_{0}^{\infty} d p p^{2} f(p) Q_{n l}(p) Q_{n^{\prime} l}(p)=\delta_{n n^{\prime}}
$$

so that coefficients of the expansion are again obtainable by quadratures,

$$
\begin{aligned}
\gamma_{l_{1} l_{2} m}^{n_{1} n_{2}}(r)= & \int_{0}^{\infty} d p_{1} d p_{2}\left[4 \pi p_{1}^{2} f\left(p_{1}\right)\right]\left[4 \pi p_{2}^{2} f\left(p_{2}\right)\right] \\
& \times \gamma_{l_{1} l_{2} m}\left(r, p_{1}, p_{2}\right) Q_{n_{1} l_{1}}\left(p_{1}\right) Q_{n_{2} l_{2}}\left(p_{2}\right) .
\end{aligned}
$$

Since the distribution function $f(p)$ will evolve during the course of an iterative solution, the associated polynomials $Q_{n l}(p)$ will also change. In principle, for a general $f(p)$ one may have to determine the $Q_{n l}(p)$ by elementary methods, such as Gram-Schmidt orthogonalization [11], starting from $Q_{00}(p)=1$. At least initially, however, the distribution $f(p)$ in the present calculation will be Gaussian and the needed polynomials are immediately found in the form of the eigenfunctions of the quantum-mechanical harmonic oscillator in three dimensions using spherical coordinates. For a Gaussian distribution of variance $\alpha / \beta$,

$$
f(p)=\frac{1}{(2 \pi \alpha / \beta)^{3 / 2}} \exp \left(-\frac{\beta p^{2}}{2 \alpha}\right),
$$

they are [12]

$$
\begin{aligned}
Q_{n l}(p)= & {\left[\frac{\Gamma\left(\frac{1}{2}(n-l)+1\right) \Gamma\left(\frac{3}{2}\right)}{\Gamma\left(\frac{1}{2}(n+l)+\frac{3}{2}\right)}\right]^{1 / 2}\left(\frac{\beta p^{2}}{2 \alpha}\right)^{l / 2} } \\
& \times L_{(n-l) / 2}^{l+1 / 2}\left(\frac{\beta p^{2}}{2 \alpha}\right),
\end{aligned}
$$

where $L_{n}^{b}(t)$ is an associated Laguerre polynomial $[11,13]$ and $\Gamma(z)$ is the gamma function. For the cases studied in Sec. IV, it will turn out in fact that the computed deviations of $f(p)$ from a Gaussian are very small, permitting retention of these polynomials, with changing $\alpha$, for the entire calculation.

In brief, the approach being taken here is a straightforward extension of the standard procedure for molecular fluids: To solve the coupled (OZ plus closure) equations (15) and (16), expand all functions in $\mathbf{r}$ space in the form

$$
\begin{aligned}
\gamma\left(\mathbf{r}, \mathbf{p}_{1}, \mathbf{p}_{2}\right)= & 4 \pi \sum_{n_{1}, n_{2}, l_{1}, l_{2}, m} \gamma_{l_{1} l_{2} m}^{n_{1} n_{2}}(r) Q_{n_{1} l_{1}}\left(p_{1}\right) Q_{n_{2} l_{2}}\left(p_{2}\right) \\
& \times Y_{l_{1} m}\left(\omega_{1}\right) Y_{l_{2} \bar{m}}\left(\omega_{2}\right)
\end{aligned}
$$

where the $z$ axis is along $\mathbf{r}$ and the summation indices satisfy the constraints [12] 


$$
\begin{gathered}
n=0,1,2,3, \ldots, \\
l=n, n-2, n-4, \ldots, 1 \text { or } 0, \\
m=0, \pm 1, \pm 2, \ldots, \pm l .
\end{gathered}
$$

The inverse of Eq. (28),

$$
\begin{aligned}
\gamma_{l_{1} l_{2} m}^{n_{1} n_{2}}(r)= & 4 \pi \int d \mathbf{p}_{1} d \mathbf{p}_{2} f\left(p_{1}\right) f\left(p_{2}\right) \gamma\left(\mathbf{r}, \mathbf{p}_{1}, \mathbf{p}_{2}\right) Q_{n_{1} l_{1}}\left(p_{1}\right) \\
& \times Q_{n_{2} l_{2}}\left(p_{2}\right) Y_{l_{1} m}^{*}\left(\omega_{1}\right) Y_{l_{2} \bar{m}}^{*}\left(\omega_{2}\right)
\end{aligned}
$$

follows from the complete orthonormality statement,

$$
\begin{aligned}
& 4 \pi \int d \mathbf{p} f(p) Q_{n l}(p) Q_{n^{\prime} l^{\prime}}(p) Y_{l m}(\omega) Y_{l^{\prime} m^{\prime}}^{*}(\omega) \\
& \quad=\delta_{n n^{\prime}} \delta_{l l^{\prime}} \delta_{m m^{\prime}} .
\end{aligned}
$$

Similarly, for functions in $\mathbf{k}$ space, set the $z$ axis along $\mathbf{k}$ and expand; e.g.,

$$
\begin{aligned}
\widetilde{\gamma}\left(\mathbf{k}, \mathbf{p}_{1}, \mathbf{p}_{2}\right)= & 4 \pi \sum_{n_{1}, n_{2}, l_{1}, l_{2}, m} \widetilde{\gamma}_{l_{1} l_{2} m}^{n_{1} n_{2}}(k) Q_{n_{1} l_{1}}\left(p_{1}\right) \\
& \times Q_{n_{2} l_{2}}\left(p_{2}\right) Y_{l_{1} m}\left(\omega_{1}\right) Y_{l_{2} \bar{m}}\left(\omega_{2}\right) .
\end{aligned}
$$

Note that the angles $\omega_{1}, \omega_{2}$ are referred to different axes in Eqs. (28) and (32). The coefficients of these expansions are not themselves mutual Fourier transforms.

\section{Iterative solution for $f(p)$}

The simplification these expansions effect is immediately reflected in the evaluation of Eq. (12) for $f(p)$. We note first that using the inversion, Eq. (30), one finds that the expansion coefficients of the dipole-dipole potential (6) are

$$
\begin{gathered}
\beta u_{110}^{11}(r)=-\frac{2 \alpha}{r^{3}}, \\
\beta u_{111}^{11}(r)=\beta u_{11-1}^{11}(r)=-\frac{\alpha}{r^{3}},
\end{gathered}
$$

which are the obvious generalizations (superscripts 11 appear, since $u$ is bilinear in $p_{1}, p_{2}$ ) of the coefficients for permanent dipoles. Then introducing the expansions of $u$ and $g$ into Eq. (12), we find immediately

$$
\frac{d}{d p} \ln \left[\frac{f(p)}{f_{0}(p)}\right]=-\left(\frac{4 \beta}{3 \alpha}\right)^{1 / 2} \sum_{n=2,4,6, \ldots} \xi_{n} Q_{n-1,1}(p),
$$

where

$$
\xi_{n}=\frac{1}{2} \rho \int d \mathbf{r} \sum_{m=-1,0,1} g_{11 m}^{n-1,1}(r) \beta u_{11 \bar{m}}^{11}(r) .
$$

Further, it is easily shown that for $n$ even,

$$
Q_{n-1,1}(p)=-\left(\frac{\alpha}{n \beta}\right)^{1 / 2} \frac{d Q_{n 0}(p)}{d p},
$$

so that Eq. (34) can be integrated to give the new fluctuatingdipole distribution as

$$
\begin{aligned}
\ln \left[\frac{f(p)}{f_{0}(p)}\right] & =\sum_{n=2,4,6, \ldots}\left(\frac{4}{3 n}\right)^{1 / 2} \xi_{n} Q_{n 0}(p) \\
& =\sum_{j=1}^{\infty}\left(\frac{2}{3 j}\right)^{1 / 2} \xi_{2 j} \hat{L}_{j}^{1 / 2}\left(\frac{\beta p^{2}}{2 \alpha}\right) .
\end{aligned}
$$

Here $\hat{L}_{j}^{1 / 2}(t)$ is the associated Laguerre polynomial renormalized to unity,

$$
\hat{L}_{j}^{l+1 / 2}(t)=\left[\frac{\Gamma(j+1) \Gamma(l+3 / 2)}{\Gamma(j+l+3 / 2)}\right]^{1 / 2} L_{j}^{l+1 / 2}(t) .
$$

More explicitly, Eq. (37) reads

$$
\begin{aligned}
\ln f(p)= & \ln f_{0}(p)+\xi_{2}\left(1-\frac{\beta p^{2}}{3 \alpha}\right)+\xi_{4}\left(\frac{5}{8}\right)^{1 / 2}\left[1-2\left(\frac{\beta p^{2}}{3 \alpha}\right)\right. \\
& \left.+\frac{3}{5}\left(\frac{\beta p^{2}}{3 \alpha}\right)^{2}\right]+\cdots
\end{aligned}
$$

As mentioned earlier, it is found by calculation that, for the cases studied in Sec. IV, $\xi_{4}$ is very small, smaller than $\xi_{2}$ by some two orders of magnitude. In these circumstances, $f(p)$ remains Gaussian to a very good approximation and its iterative recalculation simplifies to finding just its new width $\alpha$. We have

$$
\begin{aligned}
\ln f(p) & \approx-\frac{3}{2} \ln \left(\frac{2 \pi \alpha_{0}}{\beta}\right)+\xi_{2}-\left(\frac{1}{\alpha_{0}}+\frac{2}{3} \frac{\xi_{2}}{\alpha}\right) \frac{\beta p^{2}}{2} \\
& \approx-\frac{3}{2} \ln \left(\frac{2 \pi \alpha^{\prime}}{\beta}\right)-\frac{\beta p^{2}}{2 \alpha^{\prime}}
\end{aligned}
$$

where $\alpha^{\prime}$, the new $\alpha$ for the next iteration, is

$$
\alpha^{\prime}=\alpha_{0}\left(1+\frac{2}{3} \frac{\alpha_{0}}{\alpha} \xi_{2}\right)^{-1},
$$

and so, upon convergence, we have finally

$$
\alpha=\alpha_{0}\left(1-\frac{2}{3} \xi_{2}\right) .
$$

If we further note that $\xi_{2}$ is just the reduced dipole-dipole mean energy, $\xi_{2}=\beta \bar{U}_{D D} / N$, we see that Eq. (41) is precisely the iterative scheme derived by Pratt [3] and by Hळye and Stell [4] in the context of their solutions of the MSA version of this problem. It is clear that this result is in fact quite general and not restricted to the MSA and other linearized approximations.

\section{Iterative solution for $g\left(r, p_{1}, p_{2}\right)$}

With the internal degrees of freedom included in the expansion bases, the solution of the (OZ plus closure) equations for a polarizable fluid follows along a path already taken for purely molecular fluids $[14,15]$. Thus, upon introducing the full expansions (32) for $\widetilde{\gamma}$ and $\widetilde{c}$ and exploiting the orthonormality of the basis functions, Eq. (31), one finds 
that the Ornstein-Zernike equation (15) goes over into a set of matrix equations for the respective coefficients

$$
\widetilde{\gamma}_{l_{1} l_{2} m}^{n_{1} n_{2}}(k)=(-1)^{m} \rho \sum_{n_{3}, l_{3}}\left[\widetilde{\gamma}_{l_{1} l_{3} m}^{n_{1} n_{3}}(k)+\widetilde{c}_{l_{1} l_{3} m}^{n_{1} n_{3}}(k)\right] \widetilde{c}_{l_{3} l_{2} m}^{n_{3} n_{2}}(k)
$$

or, in matrix notation,

$$
\widetilde{\Gamma}_{m}(k)=(-1)^{m} \rho\left[\widetilde{\Gamma}_{m}(k)+\widetilde{C}_{m}(k)\right] \widetilde{C}_{m}(k),
$$

which have the solution

$$
\left(\begin{array}{ccc}
\widetilde{c}_{111}^{11}(k) & \widetilde{c}_{111}^{13}(k) & 0 \\
\widetilde{c}_{111}^{31}(k) & \widetilde{c}_{111}^{33}(k) & 0 \\
0 & 0 & \widetilde{c}_{221}^{22}(k) \\
0 & 0 & \widetilde{c}_{221}^{42}(k) \\
\widetilde{c}_{311}^{31}(k) & \widetilde{c}_{311}^{33}(k) & 0 \\
0 & 0 & \widetilde{c}_{421}^{42}(k)
\end{array}\right.
$$

$\left.\begin{array}{ccc}0 & \widetilde{c}_{131}^{13}(k) & 0 \\ 0 & \widetilde{c}_{131}^{33}(k) & 0 \\ \widetilde{c}_{221}^{24}(k) & 0 & \widetilde{c}_{241}^{24}(k) \\ \widetilde{c}_{221}^{44}(k) & 0 & \widetilde{c}_{241}^{44}(k) \\ 0 & \widetilde{c}_{331}^{33}(k) & 0 \\ \widetilde{c}_{421}^{44}(k) & 0 & \widetilde{c}_{441}^{44}(k)\end{array}\right)$.

Here $\widetilde{\Gamma}_{m}(k)$ and $\widetilde{C}_{m}(k)$ are, respectively, the symmetric matrices with elements $\widetilde{\gamma}_{l_{1} l_{2} m}^{n_{1} n_{2}}(k)$ and $\widetilde{c}_{l_{1} l_{2} m}^{n_{1} n_{2}}(k), n, l \geqslant m$, and $I$ is the unit matrix. The degree of these matrices is determined by the largest value of the index $m$, call it $M$, that is used in a calculation. Thus, by way of example, for $M=4$ the matrix $\widetilde{C}_{1}(k)$ is

spectively, all of order $N_{p}$ and so each with $N_{p}$ roots. The $w$ are the corresponding Gaussian weights

$$
\begin{gathered}
w(i)=\left\{t_{i}\left[\hat{L}_{N_{p}}^{1 / 2 \prime}\left(t_{i}\right)\right]^{2}\right\}^{-1}, \\
w(k)=\left\{\left(1-x_{k}^{2}\right)\left[P_{N_{p}}^{\prime}\left(x_{k}\right)\right]^{2}\right\}^{-1}, \\
w(j)=N_{p}^{-1},
\end{gathered}
$$

where prime denotes derivative. Finally, $\mathcal{P}_{l m}(x)$ in Eq. (46) is the associated Legendre function normalized to 2 .

Equation (46) and its numerical conjugate [from Eq. (28)]

$$
\begin{aligned}
\gamma\left(r, i_{1}, i_{2}, k_{1}, k_{2}, j\right)= & \sum_{n_{1}, n_{2}, l_{1}, l_{2}, m} \gamma_{l_{1} l_{2} m}^{n_{1} n_{2}}(r) Q_{n_{1} l_{1}}\left(i_{1}\right) \\
& \times Q_{n_{2} l_{2}}\left(i_{2}\right) \mathcal{P}_{l_{1} m}\left(k_{1}\right) \mathcal{P}_{l_{2} m}\left(k_{2}\right) \nu_{m} T_{m}(j)
\end{aligned}
$$

constitute separable, five-dimensional transforms. They are each executed as five successive one-dimensional transforms, following a generalized fast-transform algorithm proposed by Orszag [17]. In Eq. (50), $\nu_{0}=1$ and $\nu_{m}=2$ for $m>0$.

The OZ equation (45) effects the transformation

$$
\widetilde{c}_{l_{1} l_{2} m}^{n_{1} n_{2}}(k) \rightarrow \widetilde{\gamma}_{l_{1} l_{2} m}^{n_{1} n_{2}}(k)
$$

in Fourier transform space, while the closure relation produces the inverse transformation in direct space,

$$
\gamma_{l_{1} l_{2} m}^{n_{1} n_{2}}(r) \rightarrow c_{l_{1} l_{2} m}^{n_{1} n_{2}}(r)
$$


[combining Eq. (46) with $c=g-1-\gamma$ ]. It remains to link these two together, in a cyclic loop for the iterative solution of $\gamma$, by the Fourier transform steps

$$
c_{l_{1} l_{2} m}^{n_{1} n_{2}}(r) \rightarrow \widetilde{c}_{l_{1} l_{2} m}^{n_{1} n_{2}}(k)
$$

and the inverse

$$
\widetilde{\gamma}_{l_{1} l_{2} m}^{n_{1} n_{2}}(k) \rightarrow \gamma_{l_{1} l_{2} m}^{n_{1} n_{2}}(r)
$$

Because the angles $\omega$ associated with the conjugate coefficients are defined in different spaces (with the $z$ axis along $\mathbf{r}$ in one case and along $\mathbf{k}$ in the other) there are several parts to these Fourier transforms that are detailed in earlier publications $[14,16]$.

The final result is a computational cycle for $\gamma$ that is repeated until convergence is achieved, whereupon $g\left(\mathbf{r}, \mathbf{p}_{1}, \mathbf{p}_{2}\right)$ is known for the current dipole distribution $f(p)$. The program then returns to Eq. (37) for recalculation of $f(p)$ (actually, recalculation of the width $\alpha$ ) and a new cycle is started for $\gamma$. [The (OZ plus closure) cycle for $\gamma$ is "informed" of the new $f(p)$ through the new values of the $u$ coefficients in Eqs. (33).] The entire calculation is repeated until overall self-consistency is reached for both $f(p)$ and $g\left(\mathbf{r}, \mathbf{p}_{1}, \mathbf{p}_{2}\right)$. The iterations for $f(p)$ converge very rapidly.

\section{ELECTROSTATICS}

Several electrostatic properties of the nonpolar, polarizable liquid are computed in Sec. IV. Here we briefly review their derivation using the formalism developed above. A great amount of progress has been made in recent years in formulating an atomistic description of the electrostatic properties of fluids [18-22], in contrast to the traditional view of dielectric materials as continuous. In this section, we seek to exploit the orthogonal polynomial expansions for polarizable fluids to rederive some of these results in a compact fashion.

\section{A. Polarization density and dielectric constant}

The polarization density at point $\mathbf{r}$ in the fluid in the presence of an external electric field $\mathbf{E}^{0}(\mathbf{r})$ is defined by the canonical average

$$
\mathbf{P}(\mathbf{r})=\left\langle\sum_{i} \mathbf{p}_{i} \delta\left(\mathbf{r}-\mathbf{r}_{i}\right)\right\rangle,
$$

taken with the probability density in $Z$, Eq. (2). Equivalently, it is obtained, in component form, as the functional derivative

$$
\frac{1}{\beta} \frac{\delta \ln Z}{\delta E_{\alpha}^{0}(\mathbf{r})}=\left\langle\sum_{i} p_{\alpha i} \delta\left(\mathbf{r}-\mathbf{r}_{i}\right)\right\rangle=P_{\alpha}(\mathbf{r})
$$

where subscript $\alpha$ is $x, y$, or $z$, the Cartesian components. The response $\mathbf{P}(\mathbf{r})$ can formally be calculated to first order in the perturbing field $\mathbf{E}^{0}(\mathbf{r})$ in the form of a truncated functional Taylor-series expansion

$$
\begin{aligned}
P_{\alpha}(\mathbf{r}) & =\left.\int d \mathbf{r}^{\prime} \sum_{\beta} \frac{\delta P_{\alpha}(\mathbf{r})}{\delta E_{\beta}^{0}\left(\mathbf{r}^{\prime}\right)}\right|_{\mathbf{E}^{0}=0} E_{\beta}^{0}\left(\mathbf{r}^{\prime}\right) \\
& =\int d \mathbf{r}^{\prime} \sum_{\beta} \chi_{\alpha \beta}^{0}\left(\mathbf{r}-\mathbf{r}^{\prime}\right) E_{\beta}^{0}\left(\mathbf{r}^{\prime}\right),
\end{aligned}
$$

where

$$
\begin{aligned}
\chi_{\alpha \beta}^{0}\left(\mathbf{r}-\mathbf{r}^{\prime}\right) & =\left.\frac{1}{\beta} \frac{\delta^{2} \ln Z}{\delta E_{\beta}^{0}\left(\mathbf{r}^{\prime}\right) \delta E_{\alpha}^{0}(\mathbf{r})}\right|_{\mathbf{E}^{0}=0} \\
& =\beta\left\langle\sum_{i, j} p_{\alpha i} p_{\beta j} \delta\left(\mathbf{r}-\mathbf{r}_{i}\right) \delta\left(\mathbf{r}^{\prime}-\mathbf{r}_{j}\right)\right\rangle_{\mathbf{E}^{0}=0}
\end{aligned}
$$

is the susceptibility tensor of the system with respect to the external field. Applying the definitions of the one- and twobody distribution functions, Eqs. (8) and (9), and specializing them for zero field, we find

$$
\begin{aligned}
\chi_{\alpha \beta}^{0}(r)= & \beta \int d \mathbf{p} d \mathbf{p}^{\prime} p_{\alpha} p_{\beta}^{\prime}\left\{\rho f(p) \delta(\mathbf{r}) \delta\left(\mathbf{p}-\mathbf{p}^{\prime}\right)\right. \\
& \left.+\rho^{2} f(p) f\left(p^{\prime}\right) g\left(\mathbf{r}, \mathbf{p}, \mathbf{p}^{\prime}\right)\right\}=\left[\frac{\rho \beta}{3}\left\langle p^{2}\right\rangle \delta(\mathbf{r})\right. \\
& \left.+\beta \rho^{2} \int d \mathbf{p} d \mathbf{p}^{\prime} f(p) f\left(p^{\prime}\right) g\left(\mathbf{r}, \mathbf{p}, \mathbf{p}^{\prime}\right) p_{\alpha} p_{\alpha}^{\prime}\right] \delta_{\alpha \beta} .
\end{aligned}
$$

We may now put the $z$ axis in the direction of $\mathbf{r}$ and use the full expansion of $g\left(\mathbf{r}, \mathbf{p}, \mathbf{p}^{\prime}\right)$ [see Eq. (28)] to evaluate the remaining integrals. The result is

$$
\begin{gathered}
\chi_{x x}^{0}(r)=\chi_{y y}^{0}(r)=\alpha \rho\left[\delta(\mathbf{r})-\rho g_{111}^{11}(r)\right], \\
\chi_{z z}^{0}(r)=\alpha \rho\left[\delta(\mathbf{r})+\rho g_{110}^{11}(r)\right],
\end{gathered}
$$

where we have used $\left\langle p^{2}\right\rangle=3 \alpha / \beta$.

The transform of $\chi_{\alpha \alpha}^{0}(r)$ from Eq. (55) is

$$
\widetilde{\chi}_{\alpha \alpha}^{0}(k)=\alpha \rho+\beta \rho^{2} \int d \mathbf{p} d \mathbf{p}^{\prime} f(p) f\left(p^{\prime}\right) \widetilde{h}\left(\mathbf{k}, \mathbf{p}, \mathbf{p}^{\prime}\right) p_{\alpha} p_{\alpha}^{\prime}
$$

and expansion of $\tilde{h}\left(\mathbf{k}, \mathbf{p}, \mathbf{p}^{\prime}\right)$ as in Eq. (32), with $z$ axis along $\mathbf{k}$, similarly leads to

$$
\begin{gathered}
\widetilde{\chi}_{x x}^{0}(k)=\widetilde{\chi}_{y y}^{0}(k)=\alpha \rho\left[1-\rho \widetilde{h}_{111}^{11}(k)\right], \\
\widetilde{\chi}_{z z}^{0}(k)=\alpha \rho\left[1+\rho \widetilde{h}_{110}^{11}(k)\right] .
\end{gathered}
$$

The connection between the dielectric constant $\epsilon$ and these susceptibilities is [22]

$$
\begin{gathered}
4 \pi \lim _{k \rightarrow 0} \widetilde{\chi}_{x x}^{0}(k)=4 \pi \lim _{k \rightarrow 0} \tilde{\chi}_{y y}^{0}(k)=\epsilon-1, \\
4 \pi \lim _{k \rightarrow 0} \tilde{\chi}_{z z}^{0}(k)=\frac{\epsilon-1}{\epsilon},
\end{gathered}
$$


and so we find

$$
\begin{aligned}
& \epsilon-1=4 \pi \alpha \rho\left[1-\rho \widetilde{h}_{111}^{11}(0)\right], \\
& \frac{\epsilon-1}{\epsilon}=4 \pi \alpha \rho\left[1+\rho \widetilde{h}_{110}^{11}(0)\right] .
\end{aligned}
$$

The ratio of these gives finally

$$
\epsilon=\frac{1-\rho \widetilde{h}_{111}^{11}(0)}{1+\rho \widetilde{h}_{110}^{11}(0)}
$$

which is the direct analog (with superscripts 11) of the dielectric constant of permanent dipoles.

Other analogs are easily obtained. Thus, from the trace of $\widetilde{\chi}_{\alpha \beta}^{0}(0)$, we have

$$
\begin{gathered}
\frac{(\epsilon-1)(2 \epsilon+1)}{3 \epsilon}=4 \pi \alpha \rho\left[1+\frac{1}{3} \rho \widetilde{h}_{\Delta}^{11}(0)\right], \\
\widetilde{h}_{\Delta}^{11}(k) \equiv \widetilde{h}_{110}^{11}(k)-2 \widetilde{h}_{111}^{11}(k),
\end{gathered}
$$

while the difference of Eqs. (63) and (64) gives

$$
\begin{aligned}
& \frac{(\epsilon-1)^{2}}{\epsilon}=-4 \pi \alpha \rho^{2} \widetilde{h}_{D}^{11}(0), \\
& \widetilde{h}_{D}^{11}(k) \equiv \widetilde{h}_{110}^{11}(k)+\widetilde{h}_{111}^{11}(k) .
\end{aligned}
$$

\section{B. Reaction field of a fixed dipole}

In the absence of an external field, the polarization density from Eq. (51) of course vanishes by symmetry,

$$
\mathbf{P}(\mathbf{r})=\int d \mathbf{p} \rho^{(1)}(\mathbf{r}, \mathbf{p}) \mathbf{p}=\rho \int d \mathbf{p} f(p) \mathbf{p}=0 .
$$

If, however, we calculate the conditional polarization density at $\mathbf{r}$, given a dipole $\mathbf{p}_{0}$ at the origin, we get the induced field of the dipole $\mathbf{p}_{0}$. Define the joint distribution

$$
\begin{aligned}
\mathbf{P}\left(\mathbf{r}, \mathbf{p}_{0}\right) & =\left\langle\sum_{i \neq j} \mathbf{p}_{j} \delta\left(\mathbf{r}-\mathbf{r}_{j}\right) \delta\left(\mathbf{r}_{i}\right) \delta\left(\mathbf{p}_{0}-\mathbf{p}_{i}\right)\right\rangle_{\mathbf{E}^{0}=0} \\
& =\rho^{2} f\left(p_{0}\right) \int d \mathbf{p} f(p) g\left(\mathbf{r}, \mathbf{p}_{0}, \mathbf{p}\right) \mathbf{p} .
\end{aligned}
$$

The density of molecules with dipole $\mathbf{p}_{0}$ at the origin is $\rho f\left(p_{0}\right)$, so the conditional distribution of polarization given $\mathbf{p}_{0}$ at the origin is

$$
\mathbf{P}\left(\mathbf{r} \mid \mathbf{p}_{0}\right)=\frac{\mathbf{P}\left(\mathbf{r}, \mathbf{p}_{0}\right)}{\rho f\left(p_{0}\right)}=\rho \int d \mathbf{p} f(p) g\left(\mathbf{r}, \mathbf{p}_{0}, \mathbf{p}\right) \mathbf{p} .
$$

Once again, the full expansion of $g\left(\mathbf{r}, \mathbf{p}_{0}, \mathbf{p}\right)$ allows this integral to be readily evaluated. The result is

$$
\mathbf{P}\left(\mathbf{r} \mid \mathbf{p}_{0}\right)=\rho H_{110}\left(r, p_{0}\right)\left(\hat{\mathbf{r}} \cdot \mathbf{p}_{0}\right) \hat{\mathbf{r}}+\rho H_{111}\left(r, p_{0}\right)\left[\left(\hat{\mathbf{r}} \cdot \mathbf{p}_{0}\right) \hat{\mathbf{r}}-\mathbf{p}_{0}\right],
$$

where

$$
\begin{aligned}
H_{110}\left(r, p_{0}\right) & =\sum_{n=1,3,5, \ldots} g_{110}^{n 1}(r) Q_{n 1}\left(p_{0}\right) / Q_{11}\left(p_{0}\right) \\
& =\sum_{j=0}^{\infty} g_{110}^{2 j+1,1}(r) \hat{L}_{j}^{3 / 2}\left(\frac{\beta p_{0}^{2}}{2 \alpha}\right)
\end{aligned}
$$

and similarly

$$
H_{111}\left(r, p_{0}\right)=\sum_{j=0}^{\infty} g_{111}^{2 j+1,1}(r) \hat{L}_{j}^{3 / 2}\left(\frac{\beta p_{0}^{2}}{2 \alpha}\right) .
$$

Alternatively, Eq. (73) can be rearranged to read

$$
\mathbf{P}\left(\mathbf{r} \mid \mathbf{p}_{0}\right)=\frac{1}{3} \rho H_{\Delta}\left(r, p_{0}\right) \mathbf{p}_{0}+\frac{1}{3} \rho H_{D}\left(r, p_{0}\right)\left[3\left(\hat{\mathbf{r}} \cdot \mathbf{p}_{0}\right) \hat{\mathbf{r}}-\mathbf{p}_{0}\right],
$$

where $H_{\Delta} \equiv H_{110}-2 H_{111}$ and $H_{D} \equiv H_{110}+H_{111}$. The $j=0$ term of Eq. (76) is Pratt's result [3] in the MSA. Following Pratt, we use this distribution to get the reaction field $\mathbf{E}_{R}\left(p_{0}\right)$ produced at the origin by the polarized system,

$$
\begin{aligned}
\mathbf{E}_{R}\left(p_{0}\right) & =\int d \mathbf{r} \frac{1}{r^{3}}(3 \hat{\mathbf{r}} \hat{\mathbf{r}}-\mathbf{1}) \cdot \mathbf{P}\left(\mathbf{r} \mid \mathbf{p}_{0}\right) \\
& =\mathbf{p}_{0} \frac{2}{3} \rho \int d \mathbf{r} \frac{H_{D}\left(r, p_{0}\right)}{r^{3}}=\mathbf{p}_{0} \sum_{j=0}^{\infty} \mathcal{E}_{2 j+1} \hat{L}_{j}^{3 / 2}\left(\frac{\beta p_{0}^{2}}{2 \alpha}\right),
\end{aligned}
$$

where

$$
\mathcal{E}_{n}=\frac{2}{3} \rho \int d \mathbf{r} \frac{1}{r^{3}}\left[g_{110}^{n 1}(r)+g_{111}^{n 1}(r)\right]
$$

More explicitly, we have

$$
E_{R}\left(p_{0}\right) / p_{0}=\mathcal{E}_{1}+\mathcal{E}_{3}\left(\frac{5}{2}\right)^{1 / 2}\left(1-\frac{\beta p_{0}^{2}}{5 \alpha}\right)+\cdots,
$$

where again the leading term $\mathcal{E}_{1}$ is Pratt's result [3].

\section{RESULTS}

Using the integral equation procedures described above, the thermodynamic, dielectric, and structural properties of a system of nonpolar, polarizable Lennard-Jones molecules have been calculated for ten states for which the moleculardynamics data of Pollock and Alder [23,24] are available. The specific closure used is the optimized reference hypernetted-chain (RHNC) approximation [25-27], with the bridge function $B$ represented by just a spherically symmetric hard-sphere term

$$
B\left(\mathbf{r}, \mathbf{p}_{1}, \mathbf{p}_{2}\right) \approx B_{\mathrm{HS}}\left(r ; \sigma_{\mathrm{HS}}\right)
$$

The critical feature in this approximation is that the hardsphere diameter $\sigma_{\mathrm{HS}}$ is considered adjustable [26]; it is chosen to satisfy the optimization condition [27]

$$
\rho \int d \mathbf{r}\left[g_{000}^{00}(r)-g_{\mathrm{HS}}\left(r ; \sigma_{\mathrm{HS}}\right)\right] \frac{\partial B_{\mathrm{HS}}\left(r ; \sigma_{\mathrm{HS}}\right)}{\partial \sigma_{\mathrm{HS}}}=0,
$$


TABLE I. Computed thermodynamic properties of a nonpolar, polarizable Lennard-Jones liquid for ten states characterized by $\rho, T$, and $\alpha_{0}$ values; $\sigma_{\mathrm{HS}}$ is the corresponding hard-sphere diameter of the reference system for the optimized RHNC closure used in the calculation.

\begin{tabular}{lcccccccc}
\hline \hline State & $\rho \sigma^{3}$ & $k_{B} T / \epsilon_{\mathrm{LJ}}$ & $\alpha_{0} / \sigma^{3}$ & $\sigma_{\mathrm{HS}} / \sigma$ & $\beta \bar{U}_{D D} / N$ & $\beta U / N$ & $\beta p / \rho$ & $\partial(\beta p) / \partial \rho$ \\
\hline 1 & 0.840 & 0.700 & 0.02 & 1.0244 & -0.007 & -8.709 & -0.213 & 21.79 \\
2 & 0.844 & 0.838 & 0.02 & 1.0160 & -0.007 & -7.127 & 1.121 & 22.12 \\
3 & 0.844 & 0.820 & 0.04 & 1.0169 & -0.027 & -7.326 & 0.974 & 22.13 \\
4 & 0.840 & 0.720 & 0.05 & 1.0230 & -0.041 & -8.470 & -0.023 & 21.67 \\
5 & 0.844 & 0.832 & 0.07 & 1.0161 & -0.082 & -7.258 & 1.022 & 21.96 \\
6 & 0.200 & 2.010 & 0.10 & 0.9081 & -0.037 & -0.689 & 0.808 & 0.713 \\
7 & 0.700 & 1.970 & 0.10 & 0.9743 & -0.144 & -2.332 & 2.028 & 8.166 \\
8 & 0.840 & 0.670 & 0.10 & 1.0260 & -0.168 & -9.304 & -0.686 & 21.48 \\
9 & 0.844 & 0.836 & 0.10 & 1.0156 & -0.172 & -7.305 & 0.986 & 21.74 \\
10 & 0.840 & 0.800 & 0.14 & 1.0171 & -0.362 & -7.825 & 0.486 & 20.73 \\
\hline \hline
\end{tabular}

which minimizes the free energy and improves the internal thermodynamic consistency of the approximation. A better approximation would include at least the same coefficients of $B$ as are present in the total potential, but little is known about the higher coefficients. However, experience with systems of dipolar molecules $[16,28]$ and the relative smallness of the induced dipolar effects suggest that reasonable results can be obtained with just Eq. (80). The hard-sphere correlation functions needed for this are modeled with empirical fits $[29,30]$. The closure, Eq. (80), is the only approximation in this calculation.

Integrals over $r$ are evaluated using the trapezoidal rule on a grid of $N_{r}=1024$ points with an interval $\Delta r / \sigma=0.02$. Similarly, integrals over $k$ are evaluated using the trapezoidal rule and $N_{r}$ points, with an interval $\Delta k=\pi / N_{r} \Delta r$. The Gaussian quadratures for the expansion coefficients, Eq. (46), are carried out with $N_{p}=6$ points; increasing this to $N_{p}=10$ produces no significant changes in the computed results.

The thermodynamic properties computed are the usual internal energy $U$, pressure $p$, and isothermal compressibility $K_{T}$. For a total potential $\phi\left(\mathbf{r}, \mathbf{p}_{1}, \mathbf{p}_{2}\right)$, the internal energy is given by

$$
\begin{aligned}
\frac{\beta U}{N} & =\frac{1}{2} \rho \int d \mathbf{r} d \mathbf{p}_{1} d \mathbf{p}_{2} f\left(p_{1}\right) f\left(p_{2}\right) g\left(\mathbf{r}, \mathbf{p}_{1}, \mathbf{p}_{2}\right) \beta \phi\left(\mathbf{r}, \mathbf{p}_{1}, \mathbf{p}_{2}\right) \\
& =\frac{1}{2} \rho \int d \mathbf{r} \sum_{n_{1}, n_{2}, l_{1}, l_{2}, m} g_{l_{1} l_{2} m}^{n_{1} n_{2}}(r) \beta \phi_{l_{1} l_{2} m}^{n_{1} n_{2}}(r) .
\end{aligned}
$$

For the present calculation, this becomes

$$
\frac{\beta U}{N}=\frac{\beta \bar{U}_{0}}{N}+\frac{\beta \bar{U}_{D D}}{N}
$$

where

$$
\frac{\beta \bar{U}_{0}}{N}=\frac{1}{2} \rho \int d \mathbf{r} g_{000}^{00}(r) \beta u_{0}(r)
$$

is the Lennard-Jones contribution and

$$
\frac{\beta \bar{U}_{D D}}{N}=\frac{1}{2} \rho \int d \mathbf{r} \sum_{m=-1,0,1} g_{11 m}^{11}(r) u_{11 \bar{m}}^{11}(r)
$$

the induced dipole-induced dipole part. Similarly, the general expression for the pressure

$$
\frac{\beta p}{\rho}=1-\frac{1}{6} \rho \int d \mathbf{r} \sum_{n_{1}, n_{2}, l_{1}, l_{2}, m} g_{l_{1} l_{2} m}^{n_{1} n_{2}}(r) r \beta \phi_{l_{1} l_{2} \bar{m}}^{n_{1} n_{2}}(r)
$$

here yields

$$
\frac{\beta p}{\rho}=\frac{\beta p_{0}}{\rho}+\frac{\beta \bar{U}_{D D}}{N}
$$

where

$$
\frac{\beta p_{0}}{\rho}=1-\frac{1}{6} \rho \int d \mathbf{r} g_{000}^{00}(r) r \beta u_{0}^{\prime}(r)
$$

and the dipole-dipole increment reduces to the equivalent energy contribution. In these expressions, the prime denotes a derivative. Finally, the compressibility $K_{T}$ is obtained as

$$
\frac{\partial(\beta p)}{\partial \rho}=\frac{\beta}{\rho K_{T}}=1-\rho \int d \mathbf{r} c_{000}^{00}(r) .
$$

The computed thermodynamic results are listed in Table I, along with the values of density $\rho$, temperature $T$, and intrinsic polarizibility $\alpha_{0}$ that characterize the ten states. For completeness, we also give the hard-sphere diameter $\sigma_{\mathrm{HS}}$ that results from the RHNC optimization, Eq. (81). All quantities, along with those of Table II, are expressed in dimensionless form, using the Lennard-Jones parameters of Eq. (7) where appropriate. There are no published moleculardynamics (MD) thermodynamic data for comparisons.

The dielectric properties stemming from $\alpha_{0}$ are assembled in Table II for the same ten states. We see that the exact expansion for $f(p)$ given by Eq. (37) or (39), with coefficients $\xi_{2 j}$, is very rapidly convergent. In fact, with $\xi_{4}$ about two orders of magnitude smaller that $\xi_{2}$ in all cases, $f(p)$ remains essentially Gaussian throughout, with a width $\alpha>\alpha_{0}$ that is obtainable from $\xi_{2}$ through Eq. (42). Similarly, 
TABLE II. Computed electrostatic properties of a nonpolar, polarizable Lennard-Jones liquid for the states of Table I; comparison with molecular-dynamics (MD) results of Pollock and Alder [23,24].

\begin{tabular}{lccccccccc}
\hline \hline State & \multicolumn{1}{c}{$\xi_{2}$} & $\xi_{4}$ & $\alpha / \sigma^{3}$ & $\sigma^{3} \mathcal{E}_{1}$ & $\sigma^{3} \mathcal{E}_{3}$ & Present work & MD & Present work & MD \\
\hline 1 & -0.0068 & 0.0000 & 0.0201 & 0.2259 & -0.0000 & 0.226 & 0.22 & 1.227 & 1.23 \\
2 & -0.0069 & 0.0000 & 0.0201 & 0.2302 & -0.0000 & 0.231 & 0.23 & 1.229 & \\
3 & -0.0271 & 0.0000 & 0.0407 & 0.4438 & -0.0002 & 0.445 & 0.45 & 1.497 & \\
4 & -0.0414 & 0.0000 & 0.0514 & 0.5375 & -0.0004 & 0.540 & 0.53 & 1.646 & 1.64 \\
5 & -0.0824 & 0.0001 & 0.0739 & 0.7441 & -0.0012 & 0.748 & 0.77 & 2.003 & \\
6 & -0.0372 & 0.0006 & 0.1025 & 0.2418 & -0.0039 & 0.242 & 0.23 & 1.280 & 1.27 \\
7 & -0.1437 & 0.0011 & 0.1096 & 0.8743 & -0.0069 & 0.873 & & 2.284 & 2.24 \\
8 & -0.1675 & 0.0006 & 0.1112 & 1.0047 & -0.0033 & 1.013 & & 2.688 & 2.64 \\
9 & -0.1720 & 0.0006 & 0.1115 & 1.0286 & -0.0038 & 1.035 & 1.04 & 2.699 & \\
10 & -0.3619 & 0.0032 & 0.1738 & 1.3883 & -0.0123 & 1.397 & 1.40 & 4.150 & 3.94 \\
\hline \hline
\end{tabular}

the exact expansion for the reaction field of a fixed dipole, Eq. (77) or (79), is dominated by the leading term $\mathcal{E}_{1}$; the second term with $\mathcal{E}_{3}$, however, does make a detectable, if small, difference for larger $\alpha_{0}$ and improves the agreement with the MD data of Pollock and Alder [23,24]. As in that work, the total reaction field $E_{R}\left(p_{0}\right)$ is evaluated for unit dipole strength $p_{0} /\left(\sigma^{3} \epsilon_{\mathrm{LJ}}\right)^{1 / 2}=1$. Finally, we list the dielectric constant $\epsilon$ calculated from the $\widetilde{h}_{11 m}^{11}(0)$. Where MD data are available for comparison, the computed results in Table II are seen to be quite good.

\section{CONCLUSION}

The internal degrees of freedom of molecules in a liquid may couple with the external ones of position and orientation so that they affect and are affected by the microscopic structure of the liquid. The principal motivation for this work is the sense that these internal coordinates should be manageable in liquid state calculations in much the same way as are the orientational degrees of freedom: through expansions in special orthogonal polynomials tailored to the specific cases. When the internal variable, such as the fluctuating dipole moment $\mathbf{p}$ treated in this paper, has a distribution $f(p)$, this means polynomials orthogonal with weight function $f(p)$. Given these polynomials, one can construct an algorithm for the iterative solution of the mutually dependent microscopic liquid structure, as described by the pair distribution function $g\left(\mathbf{r}, \mathbf{p}, \mathbf{p}^{\prime}\right)$, and "internal structure," as described by the dis- tribution function $f(p)$, with no further approximations beyond that of some closure relation familiar from simple classical liquids.

We have carried out here such a program for nonpolar, polarizable fluids, generalizing for arbitrary closures the MSA solutions of Pratt [3] and Hoye and Stell [4]. By being able to go beyond the Gaussian approximation for $f(p)$ that is inherent in the MSA solution, we could demonstrate by calculation that in fact this is an excellent representation of $f(p)$ in dense systems. Other models with a classical Boltzmann factor for the internal degrees of freedom can presumably be studied in the same way, most immediately systems of polar, polarizable molecules, which Høye and Stell [4] have also solved in the mean spherical approximation.

Coupling the external degrees of freedom to internal quantum-mechanical variables in a similarly computable fashion is a more challenging task. Stratt and co-workers $[1,31]$ have formulated an elegant method to convert quantum problems such as calculating the band structure of a liquid into equivalent classical problems of determining the pair structure of liquids with artificial internal degrees of freedom and have further solved such problems in the mean spherical approximation [1,31-33]. Unfortunately for the present goals, however, this conversion makes use of the analytical "replica trick," which does not lend itself to the numerical handling required for a general closure. Whether an adaptation of their method that does allow for numerical solution is possible is an open question.
[1] R. M. Stratt, Adv. Chem. Phys. 78, 1 (1990).

[2] A partial implementation of this approach has recently been used on a system of polydisperse colloidal particles, for which the distribution of the internal degree of freedom, particle size, is fixed. See F. Lado, Phys. Rev. E 54, 4411 (1996).

[3] L. R. Pratt, Mol. Phys. 40, 347 (1980).

[4] J. S. Høye and G. Stell, J. Chem. Phys. 73, 461 (1980).

[5] See, for example, T. L. Hill, Statistical Mechanics (McGrawHill, New York, 1956), Chap. 6.

[6] J. P. Hansen and I. R. McDonald, Theory of Simple Liquids (Academic, London, 1986).
[7] C. G. Gray and K. E. Gubbins, Theory of Molecular Fluids (Clarendon, Oxford, 1984), Vol. 1.

[8] L. Blum and A. J. Torruella, J. Chem. Phys. 56, 303 (1972); L. Blum, ibid. 57, 1862 (1972).

[9] This expansion differs from the convention of Gray and Gubbins by the additional prefactor of $4 \pi$.

[10] Of equal significance from a computational viewpoint is that a relative handful of coefficients can in fact competently represent the full function of three angular variables.

[11] See, for example, G. Arfken, Mathematical Methods for Physicists (Academic, Orlando, 1985). 
[12] P. M. Morse and H. Feshbach, Methods of Theoretical Physics (McGraw-Hill, New York, 1953), p. 1662ff.

[13] Handbook of Mathematical Functions, edited by $\mathrm{M}$. Abramowitz and I. A. Stegun (Dover, New York, 1965), Chap. 22.

[14] F. Lado, Mol. Phys. 47, 283 (1982).

[15] F. Lado, E. Lomba, and M. Lombardero, J. Chem. Phys. 103, 481 (1995).

[16] F. Lado, M. Lombardero, E. Enciso, S. Lago, and J. L. F. Abascal, J. Chem. Phys. 85, 2916 (1986).

[17] S. A. Orszag, in Science and Computers, edited by G.-C. Rota (Academic, New York, 1986).

[18] G. Nienhuis and J. M. Deutch, J. Chem. Phys. 55, 4213 (1971); 56, 235 (1972); 56, 1819 (1972).

[19] M. S. Wertheim, Annu. Rev. Phys. Chem. 30, 471 (1979).

[20] G. Stell, G. N. Patey, and J. S. Høye, Adv. Chem. Phys. 48, 183 (1981).
[21] P. Madden and D. Kivelson, Adv. Chem. Phys. 56, 467 (1984).

[22] A concise source that we will follow here is Hansen and McDonald, Theory of Simple Liquids (Ref. [6]), Sec. 12.5.

[23] E. L. Pollock and B. J. Alder, Phys. Rev. Lett. 39, 299 (1977).

[24] Additional molecular-dynamics results of Pollock and Alder are listed by Pratt in Ref. [3].

[25] F. Lado, Phys. Rev. A 8, 2548 (1973).

[26] Y. Rosenfeld and N. W. Ashcroft, Phys. Rev. A 20, 1208 (1979).

[27] F. Lado, Phys. Lett. 89A, 196 (1982).

[28] P. H. Fries and G. N. Patey, J. Chem. Phys. 82, 429 (1985).

[29] L. Verlet and J. J. Weis, Phys. Rev. A 5, 939 (1972).

[30] D. Henderson and E. W. Grundke, J. Chem. Phys. 63, 601 (1975).

[31] B.-C. Xu and R. M. Stratt, J. Chem. Phys. 91, 5613 (1989).

[32] B.-C. Xu and R. M. Stratt, J. Chem. Phys. 92, 1923 (1990).

[33] Z. Chen and R. M. Stratt, J. Chem. Phys. 94, 1426 (1991). 